\title{
Psychometric Evaluation of the NORC Diagnostic Screen for Gambling Problems (NODS) for the Assessment of DSM-5 Gambling Disorder
}

Brad W. Brazeau ( $\sim$ bradley.brazeau@ucalgary.ca )

University of Calgary https://orcid.org/0000-0001-6874-5170

David C. Hodgins

University of Calgary https://orcid.org/0000-0003-2737-5200

\section{Research Article}

Keywords: Gambling disorder, DSM-5, NODS, Validation, Psychometric, Assessment

Posted Date: December 13th, 2021

DOI: https://doi.org/10.21203/rs.3.rs-1162355/v1

License: (c) (1) This work is licensed under a Creative Commons Attribution 4.0 International License.

Read Full License 


\section{Abstract}

The National Opinion Research Center (NORC) Diagnostic Screen for Gambling Problems (NODS) is one of the most used outcome measures in gambling intervention trials. However, a screen based on DSM- 5 gambling disorder criteria has yet to be developed or validated since the DSM-5 release in 2013. This omission is possibly because the criteria for gambling disorder only underwent minor changes from DSM-IV to DSM-5: the diagnostic threshold was reduced from 5 to 4 criteria, and the illegal activity criterion was removed. Validation of a measure that captures these changes is still warranted. The current study examined the psychometric properties of an online self-report past-year adaptation of the NODS based on DSM-5 diagnostic criteria for gambling disorder. Additionally, the new NODS was evaluated for how well it identifies ICD-10 pathological gambling. A diverse sample of participants $(N=$ 959) was crowdsourced via Amazon's TurkPrime. Internal consistency and one-week test-retest reliability were good. High correlations $(r=.74-.77)$ with other measures of gambling problem severity were observed in addition to moderate correlations $(r=.21-.36)$ with related but distinct constructs (e.g., gambling expenditures, time spent gambling, other addictive behaviours). All nine of the DSM- 5 criteria loaded positively on one principal component, which accounted for $40 \%$ of the variance. Classification accuracy (i.e., sensitivity, specificity, predictive power) was generally very good with respect to the PGSI and ICD-10 diagnostic criteria. Future validation studies are encouraged to establish a gold standard measurement of gambling problem severity.

\section{Main Text}

Humans have been gambling for millennia, from six-sided dice used five thousand years ago to contemporary online casinos. Nearly every culture has had a relationship with gambling, although societal acceptance has varied across time and context (Raylu \& Oei, 2004). In the United States, gambling represents an occasional pastime for most of the adult population; however, past-year prevalence of disordered gambling (0.8\%) remains high (Welte et al., 2015). Given its simultaneous popularity and potential to cause significant problems, it is critical that assessment instruments accurately identify and classify leisure, at-risk, problem, and disordered gambling.

A screening instrument based on the original DSM-III criteria for pathological gambling became widely used in both clinical and community samples. More recently, the Problem Gambling Severity Index (PGSI; Ferris \& Wynne, 2001) has gained prominence. The PGSI, which is not based on DSM diagnostic criteria, contains nine self-report items that measure gambling problem severity. Total scores are used to identify low-, moderate-, and high-risk gambling activity over the past year. The PGSI has demonstrated high correlations with other measures of gambling problem severity $(r=.83)$, as well as good internal consistency $(=.86)$, excellent specificity (1.0), and adequate sensitivity (.83; Ferris \& Wynne, 2001; Holtgraves, 2009).

The PGSI includes items that survey a range of problematic gambling characteristics, but does not offer comprehensive coverage of current DSM criteria nor yield a diagnosis. To fill this gap, the National 
Opinion Research Center (NORC) Diagnostic Screen for Gambling Problems (NODS; Gerstein et al., 1999) was developed. The NODS is a 17-item diagnostic interview based on DSM-IV criteria that assesses gambling problems over the past year. The NODS correlates highly with other measures of gambling problem severity $(r=.86)$ and moderately with log-transformed monthly gambling expenditures and number of days gambled $(r=.50)$; it has also demonstrated fair internal consistency $(=.78$; Hodgins, 2004).

In 2013, the DSM-5 update included changes to the classification and diagnosis of gambling problems (APA, 2013). Renamed gambling disorder (GD) to reduce stigma, it was reclassified as an addictive disorder given its significant overlap with substance use disorders (Petry et al., 2013).

Additionally, the illegal acts criterion was removed to reflect that it was often the last criterion endorsed by those diagnosed with DSM-IV pathological gambling; the diagnostic threshold was also reduced from 5 to 4 given the removal of a criterion. A DSM- 5 diagnosis is typically derived by administering the NODS and excluding the illegal acts question; however, no measure has been developed or validated to reflect the 2013 DSM changes.

The purpose of the current study was to validate an online self-report past-year version of the NODS to assess DSM-5 gambling disorder via examination of psychometric properties (i.e., internal consistency, test-retest reliability, convergent and divergent validity, factor structure, item response patterns, sensitivity, specificity, and classification accuracy). Additionally, this version of the NODS was evaluated for how well it identifies ICD-10 pathological gambling.

\section{Methods}

\section{Participants}

Participants were recruited via Amazon's TurkPrime (Litman et al., 2017). TurkPrime is a virtual crowdsourcing platform that allows researchers to invite users to complete brief tasks (called Human Intelligence Tasks [HITs]) in exchange for financial compensation. To meet eligibility requirements, participants had to: a) be located in the United States; b) be 18 years of age or older; c) have gambled at least once in the past year; and d) demonstrate a HIT approval rate of $25 \%$ or greater (i.e., successful completion of at least $25 \%$ of attempted HITs). It bears noting that TurkPrime samples generally report higher rates of gambling problems compared to the general population (Schluter, Kim, \& Hodgins, 2018).

Remuneration amounts on TurkPrime are typically based on the anticipated length of time it will take to complete a task. Best practices recommend a compensation rate of at least ten cents per minute (Chandler \& Shapiro, 2016). For the current study, the anticipated completion time was ten minutes in total per worker; thus, participants were initially compensated a total of US\$1.00. Mean survey completion times were greater than expected, which prompted the authors to increase worker remuneration to US\$2.00. This study, including the methods, design, and modification, was approved by the University of Calgary Conjoint Faculties Research Ethics Board (REB20-1012). 


\section{Measures}

The PGSI and NODS were administered in self-report format using a past-year reporting window. A cut score of 8 or greater on the PGSI (i.e., problem gambling) was used to categorize participants; classifications were used as references for the measurement of NODS classification accuracy. Note that there is no gold standard measurement of gambling problems; the PGSI was selected for the current study on the basis of its strong psychometric properties and frequent use in epidemiological research.

The Screener for Substance and Behavioural Addictions (SSBA) is a self-report measure that asks the same four questions as they apply to respondents' engagement with ten addictions over the past year, including gambling (Schluter et al., 2018; 2020). Response options span from 0 (none of the time) to 4 (all of the time), and total scores range from 0 to 16 for each of the ten addiction scales. Each scale has demonstrated high internal consistency $(\alpha=.89-.94)$ and moderate to high correlations with other measures of the same constructs (Schluter et al., 2018).

The Composite International Diagnostic Interview gambling module (CIDI-GM) contains 17 yes/no questions that correspond to the four diagnostic criteria for pathological gambling in the International Statistical Classification of Diseases and Related Health Problems (ICD), tenth revision (World Health Organization [WHO], 2004). Participants that met all four criteria were classified as such as a reference point for measuring classification accuracy of the NODS for ICD-10 criteria. The CIDI-GM was administered in online self-report format along with the other measures included.

The Patient Health Questionnaire-9 (PHQ-9; Kroenke et al., 2001) was included to assess discriminant validity. This self-report instrument contains nine questions that assess depressive symptoms over the past two weeks. Response options range from 0 (not at all) to 3 (nearly every day) and yield a total score between 0 and 27. The PHQ-9 has shown good internal consistency ( $\alpha=.89$; Kroenke et al., 2001). Participants who endorsed thoughts that they would be better off dead or hurting themselves were directed to an automated response at the end of the survey that encouraged participants to consult a resource (e.g., family physician) or contact a crisis helpline via phone numbers provided to them.

\section{Procedure}

Advertisements were displayed in TurkPrime to individuals that met eligibility criteria including IP addresses located in the United States. Eligible respondents were redirected to Qualtrics to complete the first part of the survey.

Part one: Screening. A virtual private network (VPN) block and reCAPTCHA system were implemented within part one to prevent the enrollment of ineligible and fake participants, respectively. Several demographic survey questions were then asked to crosscheck with TurkPrime filters and gather descriptive information from the sample. Participants were also asked to estimate the average number of hours they have gambled per month and per gambling session over the last three months, as well as the average net number of dollars they won or lost per month and per gambling session. These three-month 
retrospective self-report questions were adapted from the Gambling Participation Instrument (GPI; Williams et al., 2017).

Best practices recommend dual screening of participants recruited from platforms such as TurkPrime (Kim \& Hodgins, 2017; Schluter, Kim, \& Hodgins, 2018). To that end, two randomly selected PGSI questions were presented prior to the demographic questions, in addition to the full PGSI at the end of part one. Only individuals who met eligibility criteria and had matching PGSI responses were permitted to continue with the main survey (part two) immediately. Regardless, all participants who completed the first part were automatically compensated US\$0.60.

Part two: Main survey. The main survey consisted of the NODS, CIDI-GM, SSBA, and PHQ-9. Participants who completed part two were manually compensated a bonus of US\$0.60 and invited to complete the one-week follow-up.

Part three: One-week follow-up. The one-week follow-up comprised the readministered NODS in addition to questions asking if participants were engaged and honest in their survey responses. Participants who completed part three were automatically credited with an additional US\$0.80.

\section{Data Analysis}

Data analysis covered three domains: reliability, validity, and classification accuracy. Test-retest reliability was assessed with the intraclass correlation coefficient (ICC) using a two-way mixed model to measure absolute agreement. Internal consistency was assessed with Cronbach's alpha. Correlational analysis were used to evaluate convergent and divergent validity by calculating Pearson's correlation coefficients between the NODS- 5 and measures of gambling problem severity, monthly gambling expenditures, hours per month spent gambling, depressive symptoms, and severity of concurrent addiction symptoms. The construct validity assessment entailed a principal components analysis using principal component extraction with promax rotation. Item response patterns on the PGSI and SSBA gambling scale were grouped by DSM-5 diagnostic severity (i.e., number of criteria met). Crosstabs were used to assess classification accuracy based on the PGSI and ICD-10 diagnostic criteria for pathological gambling. Sensitivity, specificity, positive predictive power, and negative predictive power were calculated from these crosstabs. Finally, percent agreement with the NODS- 5 was calculated for the PGSI and CIDIGM. All analyses were conducted with and without data from participants who did not endorse honest and attentive responses to survey questions; however, this did not significantly alter any results.

\section{Results}

All statistical analyses were conducted with R and RStudio (R Core Team, 2020). In total, 959 participants with a mean age of $39.4(M d n=37, S D=12.1)$ were recruited via TurkPrime from February to June 2021. Sample characteristics are provided in Tables 1 and 2. The one-week follow-up rate (50.8\%) was much lower than would normally be expected from crowdsourcing platforms (Kim \& Hodgins, 2017) despite 
attempts to increase retention (e.g., increased compensation, reduced HIT approval rate requirement) so follow-up invitations were discontinued for the final 315 participants.

-- TABLE 1 -

-- TABLE 2 -

\section{Reliability}

The mean NODS-5 score was $3.0(S D=2.5, M d n=2$, mode $=1$, range $=0-10)$. Of the 959 participants, $33 \%$ were low risk (score $=0-1$ ), $33 \%$ were subthreshold (score $=2-3$ ), $17 \%$ met criteria for mild GD $($ score $=4-5), 10 \%$ met criteria for moderate GD (score $=6-7)$, and $7 \%$ met criteria for severe GD (score $=$ 8-9). Internal consistency of the NODS-5 ( = .88) was good, and one-week test-retest reliability (ICC = .84, $p<.001$ ) was also good (Cicchetti, 1994). NODS-5 scores at follow-up (mean $=2.6, S D=2.2, M d n=2)$ were slightly lower than baseline scores, $t(325)=2.84, p=.005$; this change is indicative of regression to the mean and is expected given that only a subsample was retested.

\section{Validity}

Table 3 displays the Pearson correlations between the NODS-5 and validation measures. Convergent validity was strong, evidenced by high correlations with other direct measures of gambling severity ( $r=$ .74- .77). As expected, moderate correlations were observed between the NODS-5 and measures of depression, other addictions, gambling expenditures, and hours spent gambling $(r=.21-.36)$, which are indicative of divergent validity. Item response patterns for direct measures are provided in Table 4 and broken down by NODS- 5 classification.

- TABLE 3 -

- TABLE 4 -

The nine DSM-5 diagnostic criteria, based on the sixteen NODS-5 questions, were subjected to a principal components analysis to examine factor structure and establish construct validity. Results demonstrated a robust principal component that accounted for $40 \%$ of the variance and positively correlated with all nine criteria, which is indicative of a unitary construct. Two components had eigenvalues greater than one and together accounted for $51 \%$ of the variance. Promax rotated factor loadings for these two components are provided in Table 5. Five criteria loaded on Component A, which reflects cognitiveaffective aspects of problematic gambling. In contrast, the four criteria that loaded on Component $B$ reflect behavioural aspects.

- TABLE 5 -

\section{Classification Accuracy}


Classification accuracy of the NODS-5 based on DSM-5 and ICD-10 diagnostic criteria was evaluated with the PGSI and CIDI-GM categorizations, respectively (see Table 6). The NODS-5 classified more individuals as engaging in disordered gambling (34\%) compared to the PGSI (25\%) and fewer compared to the CIDIGM (39\%). Percent agreement with the NODS-5 was $83 \%$ for the PGSI and $78 \%$ for the CIDI-GM.

Sensitivity, specificity, and positive and negative predictive power of the NODS-5 are provided in Table 7.

- TABLE 6 -

-- TABLE 7 --

\section{Discussion}

The results of this study support the use of a DSM-5 self-report adaptation of the NODS in a community sample of crowdsourced individuals who gamble. Internal consistency and one-week test-retest reliability were both good. High correlations were observed between the NODS-5 and other direct measures of gambling problem severity (i.e., PGSI, SSBA gambling scale); moderate correlations were observed between the NODS- 5 and other validation measures (i.e., gambling expenditures, time spent gambling, depressive symptoms, SSBA non-gambling scales). While one might predict that gambling hours and expenditures would be more highly correlated with the NODS-5, moderate correlations are to be expected given the different timeframes (past year versus past three months) and distinct constructs (gambling behaviours versus diagnostic problem severity).

All nine of the DSM- 5 criteria loaded positively on the principal component, which accounted for $40 \%$ of the variance; this suggests that the NODS- 5 measures a single construct. Two components with eigenvalues greater than one roughly represented cognitive and behavioural symptoms of GD, which is consistent with a cognitive-behavioural theoretical formulation of gambling problems.

Classification accuracy of the NODS- 5 was generally very good with reference to the PGSI and CIDI-GM. The NODS- 5 classified more individuals as disordered compared to the PGSI and fewer compared to the CIDI-GM. Importantly, the lack of a gold standard measurement of gambling problem severity obfuscates possible conclusions. Our results should be interpreted considering this caveat.

Those familiar with TurkPrime will recognize our HIT approval rate cut-off $(25 \%)$ as low, which can raise concerns about the quality of data collected. Indeed, best practices recommend excluding participants with HIT approval rates lower than 95\% (Kim \& Hodgins, 2017; Schluter, Kim, \& Hodgins, 2018) because a low approval rate implies the associated TurkPrime worker has provided unusable data to a large proportion of prior HITs. Unfortunately, users with extremely high approval rates are much less common; the use of a low approval rate was necessary to ensure timely recruitment of participants. Despite these considerations, our adherence to other best practices (e.g., remuneration, multi-step surveys, validation procedures, dual screening) appears to have mitigated potential consequences of a low approval rate cutoff. For example, filtering out participants who admitted to responding dishonestly or inattentively did not 
significantly alter any findings. Finally, researchers have demonstrated that addiction populations on TurkPrime generally provide high quality data (Kim \& Hodgins, 2017).

\section{Conclusion}

The NODS- 5 represents a reliable and valid measure of gambling problem severity, based on DSM-5 diagnostic criteria, when administered in online self-report format to a diverse crowdsourced sample of community gamblers. Future validation studies are encouraged to establish a gold standard measurement of gambling problem severity for the psychometric evaluation of classification accuracy.

\section{References}

American Psychiatric Association (2013). Diagnostic and statistical manual of mental disorders (5th edn.). Washington, DC: American Psychiatric Publishing, Inc.

Chandler, J., \& Shapiro, D. (2016). Conducting clinical research using crowdsourced convenience samples. Annual Review of Clinical Psychology, 12(1), 53-81.

Cicchetti, D. V. (1994). Guidelines, Criteria, and Rules of Thumb for Evaluating Normed and Standardized Assessment Instruments in Psychology. Psychological Assessment, 6(4), 284-290.

Currie, S. R., Hodgins, D. C., \& Casey, D. M. (2013). Validity of the problem gambling severity index interpretive categories. Journal of Gambling Studies, 29(2), 311-327.

Ferris, J. and Wynne, H. (2001). The Canadian Problem Gambling Index: Final report. Ottawa, ON: Canadian Centre on Substance Abuse.

Gerstein, D., Hoffmann, J., Larison, C., Engelman, L., Murphy, S., \& Palmer, A. (1999). Gambling Impact and Behavior Study. National Gambling Impact Study Commission Report, Exec-2.

Hodgins, D. C. (2004). Using the NORC DSM Screen for Gambling Problems as an outcome measure for pathological gambling: Psychometric evaluation. Addictive Behaviors, 29(8), 1685-1690.

Hodgins, D. C., Stea, J. N., \& Grant, J. E. (2011). Gambling disorders. The Lancet, 378(9806), 1874-1884.

Holtgraves, T. (2009). Evaluating the Problem Gambling Severity Index. Journal of Gambling Studies, 25(1), 105-120.

Kim, H. S., \& Hodgins, D. C. (2017). Reliability and validity of data obtained from alcohol, cannabis, and gambling populations on Amazon's Mechanical Turk. Psychology of Addictive Behaviors, 31(1), 85-94.

Kroenke, K., Spitzer, R. L., \& Williams, J. B. W. (2001). The PHQ-9: Validity of a brief depression severity measure. Journal of General International Medicine, 16(02), 606-613. 
Litman, L., Robinson, J., \& Abberbock, T. (2017). TurkPrime.com: A versatile crowdsourcing data acquisition platform for the behavioral sciences. Behavior Research Methods, 49(2), 433-442.

Petry, N. M., Blanco, C., Stinchfield, R., \& Volberg, R. (2013). An empirical evaluation of proposed changes for gambling diagnosis in the DSM-5. Addiction, 108(3), 575-581.

R Core Team. (2020). R: A language and environment for statistical computing. R Foundation for Statistical Computing. http://www.R-project.org

Raylu, N., \& Oei, T. P. (2004). Role of culture in gambling and problem gambling. Clinical Psychology Review, 23(8), 1087-1114.

Schluter, M. G., Hodgins, D. C., Wolfe, J., \& Wild, T. C. (2018). Can one simple questionnaire assess substance-related and behavioural addiction problems? Results of a proposed new screener for community epidemiology. Addiction, 113(8), 1528-1537.

Schluter, M. G., Hodgins, D. C., Thege, B. K., \& Wild, T. C. (2020). Predictive utility of the brief Screener for Substance and Behavioral Addictions for identifying self-attributed problems. Journal of Behavioral Addictions, 9(3), 709-722.

Schluter, M. G., Kim, H. S., \& Hodgins, D. C. (2018). Obtaining quality data using behavioral measures of impulsivity in gambling research with Amazon's Mechanical Turk. Journal of Behavioral Addictions, 7(4), 1122-1131.

Welte, J. W., Barnes, G. M., Tidwell, M.-C. O., Hoffman, J. H., \& Wieczorek, W. F. (2015). Gambling and problem gambling in the United States: Changes between 1999 and 2013. Journal of Gambling Studies, $31(3), 695-715$.

Williams, R. J., Volberg, R. A., Stevens, R. M. G., Williams, L. A., \& Arthur, J. N. (2017). The definition, dimensionalization, and assessment of gambling participation. Report Prepared for the Canadian Consortium for Gambling Research, 1-157.

World Health Organization. (2004). ICD-10: International statistical classification of diseases and related health problems (10th ed.). World Health Organization.

\section{Tables}


Table 1

Sample Demographics $(N=959)$

Demographic variables

Descriptive statistics

Sex

Male

503

52.6

Female

452

47.1

Non-binary

3

0.3

Education

Some post-secondary or greater

751

78.3

Employment

Employed full-time or part-time

703

73.3

Unemployed

76

7.9

Annual household income

Under $\$ 30,000$

172

17.9

$\$ 30,000$ to $\$ 99,999$

559

58.3

$\$ 100,000$ and over

228

23.8

Relationship status

Married or common-law

452

47.3

Single

383

40.0

Household members

Live with spouse or common law partner

549

57.2

Live with child(ren) under 18

248

25.9

Live alone

196

20.4

Residential area

Urban

621

64.8

Rural

323

33.7

Ethnicity

White 


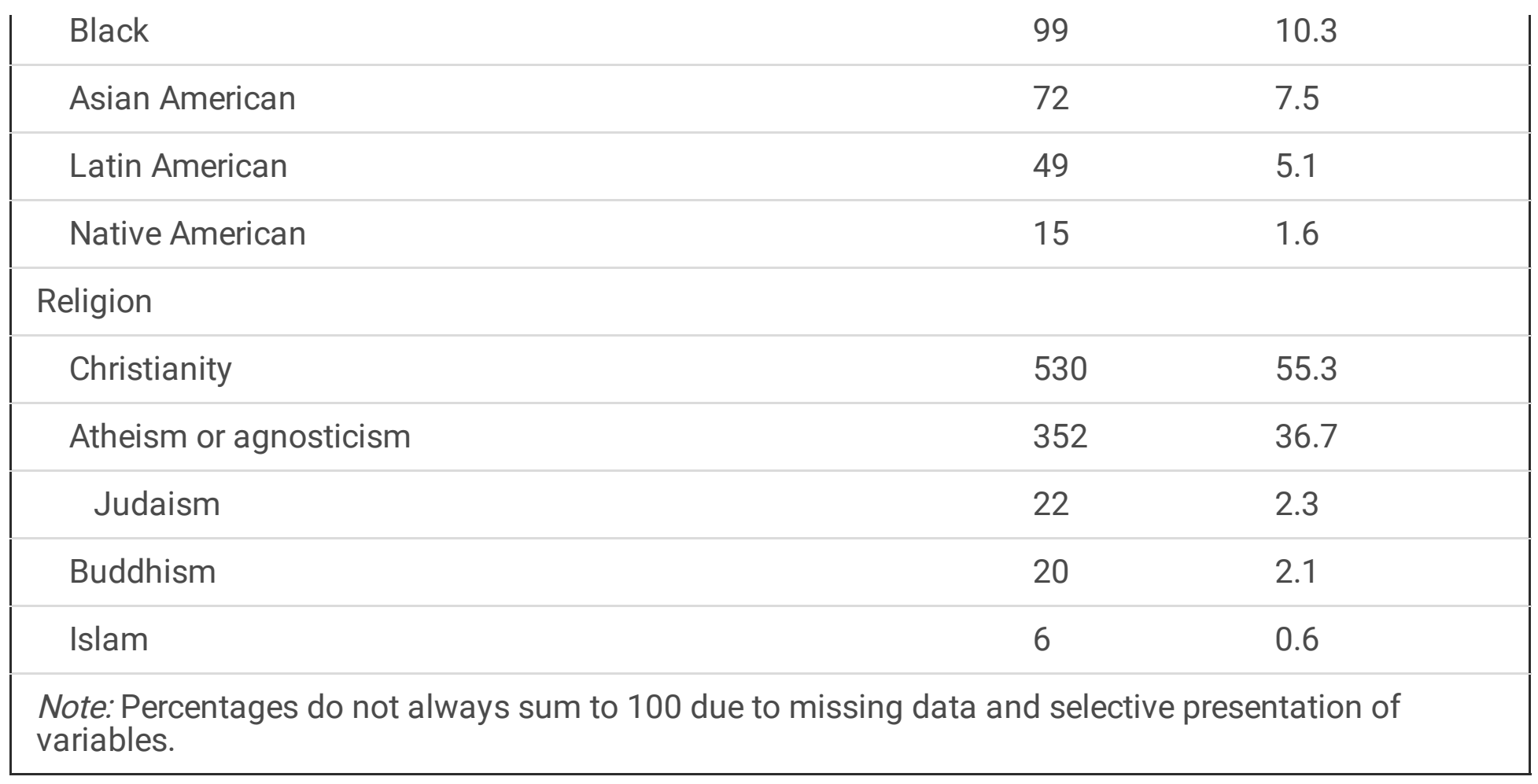


Table 2

Primary Measures and Descriptive Statistics ( $N=959)$

Primary measures Descriptive statistics

\begin{tabular}{|llll|} 
& $M$ & $M d n$ & $S D$ \\
\hline PGSI & 6.03 & 4 & 6.53 \\
\hline NODS & 3.07 & 2 & 2.59 \\
\hline NODS-5 & 3.00 & 2 & 2.46 \\
\hline
\end{tabular}

Hours spent gambling

Per session

2.18

1

2.22

Per month

18.60

6

25.60

Dollars spent gambling

Per session

104.21

25

269.11

Per month

560.28

100

1623.34

SSBA

Gambling

3.14

2

3.77

Alcohol

2.60

1

3.58

Tobacco

2.70

0

4.65

Cannabis

1.62

0

3.40

Cocaine

0.47

0

1.94

Sex

1.82

0

3.26

Video gaming

2.69

1

3.59

Overeating

3.57

2

3.95

Overworking

2.42

0

3.63

Shopping

2.74

2

3.31

PHQ-9

6.49

5

5.77

Note: NODS: National Opinion Research Center Diagnostic Screen for Gambling Problems; PGSI: Problem Gambling Severity Index; PHQ: Patient Health Questionnaire; SSBA: Screen for Substance and Behavioural Addictions. 
Table 3

Correlations between Primary Measures and the NODS-5 $(N=959)$

Primary measures

$r$

PGSI .74

Hours spent gambling

Per session .30

Per month .24

Dollars spent gambling

Per session .23

Per month

SSBA

Gambling

.72

Shopping .36

Cocaine .30

Sex .30

Video gaming .29

Tobacco .28

Overworking .28

Overeating .27

Cannabis .26

Alcohol .24

PHQ-9

.32

Note: All $p<.001$.

NODS: National Opinion Research Center Diagnostic Screen for Gambling Problems; PGSI: Problem Gambling Severity Index; PHQ: Patient Health Questionnaire; SSBA: Screen for Substance and Behavioural Addictions. 
Table 4

Mean Scores on Measures of Gambling Problems by NODS-5 Classification ( $N=959)$

Measures Classification

\begin{tabular}{llllll|} 
& $\begin{array}{l}\text { Low Risk } \\
(0-1)\end{array}$ & $\begin{array}{l}\text { Subthresh-old } \\
(2-3)\end{array}$ & $\begin{array}{l}\text { Mild GD } \\
(4-5)\end{array}$ & $\begin{array}{l}\text { Moderate GD } \\
(6-7)\end{array}$ & $\begin{array}{l}\text { Severe GD } \\
(8-9)\end{array}$ \\
\hline PGSI & 2.08 & 4.13 & 7.51 & 14.51 & 18.00 \\
\hline $\begin{array}{l}\text { SSBA: } \\
\text { Gambling }\end{array}$ & 0.92 & 2.01 & 4.31 & 7.37 & 10.04 \\
\hline
\end{tabular}

Note: GD: gambling disorder; NODS: National Opinion Research Center Diagnostic Screen for Gambling Problems; PGSI: Problem Gambling Severity Index; PHQ: SSBA: Screen for Substance and Behavioural Addictions.

Table 5

Promax Rotated Factor Loadings for the NODS-5 ( $N=959)$

DSM-5 criterion

Component

A B

Loss of control 6/7

.38

Dishonesty $11 / 12$

.35

Preoccupation 1 .33

Tolerance 3 .33

Escape from distress 8/9 .27

Financial bailout 17 .57

Life problems 14/15/16

.52

Failed quit attempts $4 / 5$ .42

Chasing losses 10

Note: NODS: National Opinion Research Center Diagnostic Screen for Gambling Problems; PGSI: Problem Gambling Severity Index. 
Table 6

Classification Accuracy of the NODS-5 ( $N=959)$

\begin{tabular}{|lllllll|}
\hline NODS-5 classification & \multicolumn{4}{l}{ PGSI classification } & Total & \multicolumn{3}{l|}{ CIDI classification } & Total \\
& No & Yes & & No & Yes & \\
\hline No (does not meet criteria) & 595 & 38 & 633 & 503 & 131 & 634 \\
\hline Yes (does meet criteria) & 121 & 205 & 326 & 79 & 246 & 325 \\
\hline Total & 716 & 243 & & 582 & 377 & \\
\hline
\end{tabular}

Note: CIDI: Composite International Diagnostic Interview; GD: gambling disorder; NODS: National Opinion Research Center Diagnostic Screen for Gambling Problems. 


\section{Table 7}

Sensitivity, Specificity, and Predictive Power of the NODS-5 ( $N=959)$

NODS-5 measurement Comparison

\begin{tabular}{lll} 
& PGSI & CIDI-GM \\
\hline Sensitivity 12 & $84 \%$ & $65 \%$ \\
\hline Specificity & $83 \%$ & $86 \%$ \\
\hline Positive predictive power & $63 \%$ & $76 \%$ \\
\hline Negative predictive power & $94 \%$ & $79 \%$
\end{tabular}

Note: CIDI-GM: Composite International Diagnostic Interview, gambling module; PGSI: Problem Gambling Severity Index. 